\title{
Excitation Analysis of Transverse Electric Mode Rectangular Waveguide
}

\author{
M. Reza Hidayat ${ }^{\text {a, }}$, Mohamad Hamzah Zamzam a, Salita Ulitia Prini ${ }^{\text {b }}$ \\ ${ }^{a}$ Department of Electrical Engineering \\ Universitas Jenderal Achmad Yani \\ $J l$. Terusan Jenderal Sudirman \\ Cimahi, Indonesia \\ ${ }^{b}$ Research Center for Electronics and Telecommunication \\ Indonesian Institute of Sciences \\ Kampus LIPI Jl. Sangkuriang Gedung 20 \\ Bandung, Indonesia
}

\begin{abstract}
A waveguide is a transmission medium in the form of a pipe and is made from a single conductor. A waveguide has the function of delivering electromagnetic waves with a frequency of $300 \mathrm{MHz}-300 \mathrm{GHz}$ and is able to direct the waves in a particular direction. In its development, a waveguide can be used as a filter. A filter consists of several circuits designed to pass signals that are generated at a specific frequency and attenuate undesired signals. One type of filter that can pass a signal in a particular frequency range and block signals that are not included in that frequency range is a bandpass filter. In this article, we study a rationing analysis on rectangular waveguide using TEmn mode followed by an implementation of a bandpass filter in the frequency range of 3.3-3.5 GHz for S-Band Wireless Broadband and Fixed Satellite. The observation process is done by shifting the position of the connector (power supply) as much as five times the shift to get the results as desired. Based on the analysis of the simulation process using Ansoft HFSS software, it is observed that the optimized results of the rectangular waveguide mode TE10 were obtained at a distance between connectors of $30 \mathrm{~mm}$ with a cut-off frequency of $3.3 \mathrm{GHz}$, the value of the return loss parameter of $-34.442 \mathrm{~dB}$ and an insertion loss of $-0.039 \mathrm{~dB}$. Whereas, the optimized TE20 mode can be obtained at a distance of $70 \mathrm{~mm}$ between connectors, with a cut-off frequency of $3.5 \mathrm{GHz}$, the value of the return loss parameter of $-28.718 \mathrm{~dB}$ and an insertion loss of 0.045. The measurement of TE10 mode in our Vector Network Analyzer (VNA) shows a cut-off frequency of $3.2 \mathrm{GHz}$, with a value of the return loss of $-18.73 \mathrm{~dB}$ and an insertion loss of $-2.70 \mathrm{~dB}$. Meanwhile, a measurement of TE20 mode results in a cutoff frequency of $3.2 \mathrm{GHz}$, with a value of the return loss of $-5.89 \mathrm{~dB}$ and an insertion loss of $-4.31 \mathrm{~dB}$.
\end{abstract}

Keywords: Filter, Frequency, Insertion Loss, Rectangular Waveguide, Return Loss.

\section{INTRODUCTION}

In the field of telecommunication, the process of delivering information from the sender to the receiver requires a transmission media. Such transmission media can be physical and non-physical. Physical transmission media is a medium that has physical forms such as cables, waveguides, or optical fibers. In contrast, non-physical media is a medium that has no physical form, such as microwave or radio waves. Waveguide [1], [2] is a transmission media with a pipe-like shape and made of a single conductor material that is partly contained in the dielectric. A waveguide has the function of delivering electromagnetic waves with a frequency of $300 \mathrm{MHz}-$ $300 \mathrm{GHz}$ and directing the waves in a certain direction.

An electromagnetic wave is a wave that can carry electrical and magnetic energy content without the needs of a medium or often called electromagnetic radiation [3], [4]. In electromagnetic waves, the electric field (E) is always perpendicular to the magnetic field (B), which both go towards the direction of the propagation. There are two modes of wave propagation in a waveguide: TE

\footnotetext{
* Corresponding Author.

Email: mreza@lecture.unjani.ac.id

Received: January 03, 2020 ; Revised: February 17, 2020

Accepted: March 06, 2020 ; Published: August 31, 2020

(C) 2020 PPET - LIPI
}

(Transverse Electric) and TM (Transverse Magnetic) [5], [6].

In the $\mathrm{TE}_{\mathrm{mn}}$ mode, the electric fields are transversal to the propagation direction of $E_{z}=0$, and the magnetic fields are longitudinal against the propagation direction. It should be noted that $\mathrm{m}$ and $\mathrm{m}$ should not be simultaneously zero. The value of $m=n=0$, resulting in a TEM mode wave. Whereas, there is no TEM mode in a waveguide.

A waveguide can be used as a filter. A filter is a device designed to pass a signal at a certain frequency and attenuate the signal at undesired frequency. One type of filter that can pass a signal within a specific frequency range and block a signal that is not included in that frequency range is the bandpass filter [7], [8].

On connection with the transmission media waveguide, analysis of comparison between the use of dielectric and without dielectric material in the performance of waveguide design has been conducted previously in [9] and [10]. A design of coaxial transition to waveguide $\mathrm{Wg} 8$ with range frequency of $1.2-3.0 \mathrm{GHz}$ was demonstrated in [9], while a design and performance analysis of the bandpass filter for Ultra wide-band Ground Penetrating Radar (GPR) applications in the frequency range $2-2.5 \mathrm{GHz}$ was studied in [10].

Inspired by the previous research [11]-[14], the research objective is to design a filter using rectangular 
waveguide without dielectric material by firstly analyzing the note on the TEmn rectangular waveguide mode. Afterward, an implementation of a bandpass filter in the frequency range of $3.3-3.5 \mathrm{GHz}$ for $\mathrm{S}$-Band Wireless Broadband and Fixed Satelite will be presented. The design and implementation done by observing changes that occur in the cut-off frequency, return loss, and insertion loss by observing the effect of distance between connectors separated symmetrically from the center of the waveguide.

\section{Proposed Method}

The research method used in this research is to firstly design and simulate the note-making in a rectangular waveguide using $\mathrm{TE}_{\mathrm{mn}}$ mode and then implement the simulation into a bandpass filter to determine the cut-off frequency, return loss and insertion loss. The simulation is carried out using Ansoft HFSS, and the validation of the simulation is measured using VNA.

The scenario of the designing method of rectangular waveguide in the simulation stage are as follows:

1. Design a $\mathrm{TE}_{\mathrm{mn}}$ rectangular waveguide mode using SMA connectors.

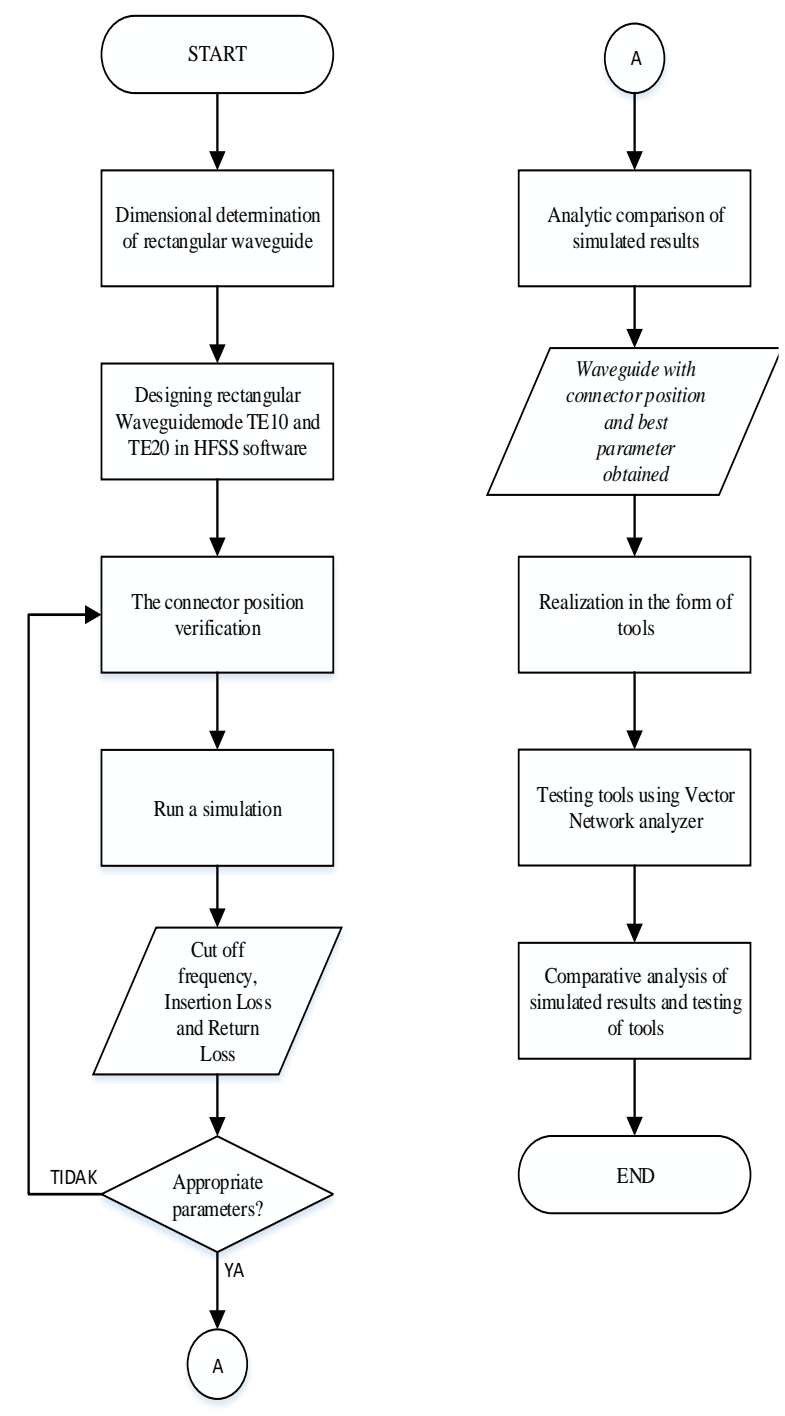

Figure 1. Flowchart Research
2. Shift the position of the connectors and observe the simulation result of $\mathrm{TE}_{\mathrm{mn}}$ rectangular waveguide mode.

3. Compare the $\mathrm{TE}_{\mathrm{mn}}$ mode simulation results.

4. Device fabrication and measurement using Vector Network Analyzer.

5. Analyze the comparison of simulation and measurement results.

Figure 1 explains the flow of the process done on this research from start to finish. The process of research is done by determining the dimension of the waveguide, designing the $\mathrm{TE}_{10}$ and $\mathrm{TE}_{20}$ rectangular waveguide in the HFSS software, shifting the position of the connector, and running the simulation. The shifting of the connector position is done to get the appropriate parameters. After that, we analyze all simulation results to obtain waveguide the best position of the connectors. The optimized waveguide is then fabricated and measured using Vector Network Analyzer (VNA). Lastly, the simulation and measurement results are compared.

\section{RESUlTS AND DISCUSSION}

\section{A. Results of TEmn Mode Rectangular Waveguide Design Using SMA Connector}

The rectangular waveguide that is used for the simulation is of type WR 284 with a width of $72 \mathrm{~mm}$, a height of $34 \mathrm{~mm}$, an outer width of $76 \mathrm{~mm}$, an outer height of $38 \mathrm{~mm}$ and a length of $100 \mathrm{~mm}$. For the enclosure dimension, the width is $76 \mathrm{~mm}$, the height is 38 $\mathrm{mm}$, the thickness is $2 \mathrm{~mm}$, and the waveguide length is $150 \mathrm{~mm}$. The material used for the rectangular waveguide and the cover is aluminum.

Figures 2 and 3 show the design using SMA connectors based on the $\mathrm{x}$-axis with a rectangular waveguide length of $100 \mathrm{~mm}$. The positions of the connector are located at $65 \mathrm{~mm}$ and $35 \mathrm{~mm}$. The center point is located at $50 \mathrm{~mm}$. Thus, the distance of the connectors from the center point is $15 \mathrm{~mm}$. Figure 2 shows the result of the $\mathrm{TE}_{10}$ mode design, and Figure 3 shows the result of the $\mathrm{TE}_{20}$ mode design. In Figures 2 and 3 , the direction of wave propagation is towards $(+x)$ indicated with a green line, while the $y$-axis (red line) and $\mathrm{z}$-axis (blue line) are the directions of magnetic and electric fields, respectively.

Figure 4 shows the graph of simulated results by using SMA connectors. At the initial position of the connector placed based on the $\mathrm{x}$-axis at position $65 / 35$, the simulation is done to know how well the performance when the rectangular waveguide uses the SMA connectors. The cut-off frequency in this mode can be calculated using (1):

$$
f c=\frac{c}{2 \pi}\left(\frac{m \pi}{a}+\frac{n \pi}{b}\right)
$$

with $m$ and $n$ are the order of the TE mode, $a$ and $b$ are the width and the height of the waveguide, respectively. As seen in the simulation results in Figure 4, frequency cut-off is shifted after using the connectors. In the $\mathrm{TE}_{10}$ mode, the cut-off frequency is shifted from $3.7 \mathrm{GHz}$ to $3.3 \mathrm{GHz}$. The value of the other parameters also becomes very good, with a return loss of $-34.442 \mathrm{~dB}$ and an 
insertion loss of $-0.039 \mathrm{~dB}$. In the TE20 mode, the cut off frequency was previously $3.6 \mathrm{GHz}$ and then shift to 3.4 $\mathrm{GHz}$. However, the values of the return loss of -17.953 $\mathrm{dB}$ and the insertion loss of $-0.109 \mathrm{~dB}$ are not better than before. The value of the cut-off frequency is approaching the expected frequency range of 3.3-3.5 GHz.

\section{B. Results of $\mathbf{T E}_{\mathrm{mn}}$ Mode Rectangular Waveguide Design with Observation on Sliding Connector Position}

The results of the $\mathrm{TE}_{\mathrm{mn}}$ rectangular waveguide mode design by performing a connector shifting position can be

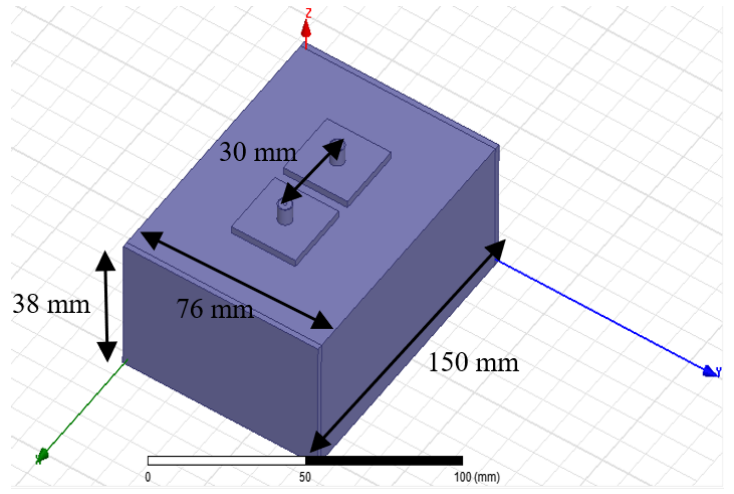

Figure 2. Rectangular waveguide design using connectors with position at $65 / 35$ ( $\mathrm{TE}_{10}$ mode).

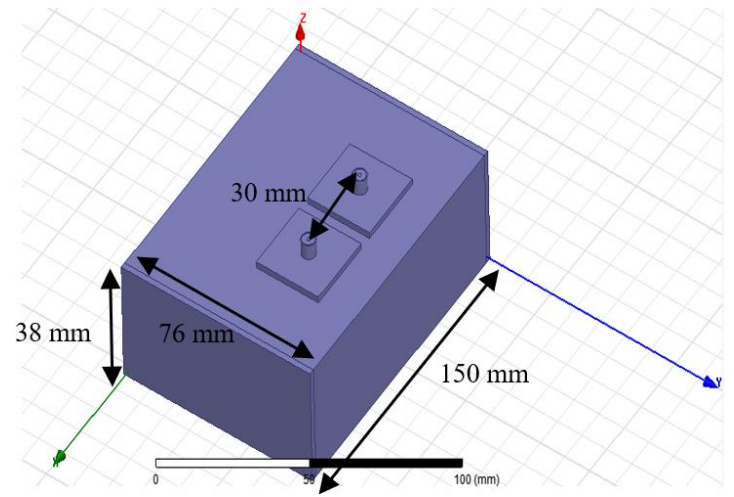

Figure 3. Rectangular waveguide design using connectors with position at $65 / 35\left(\mathrm{TE}_{20}\right.$ mode $)$

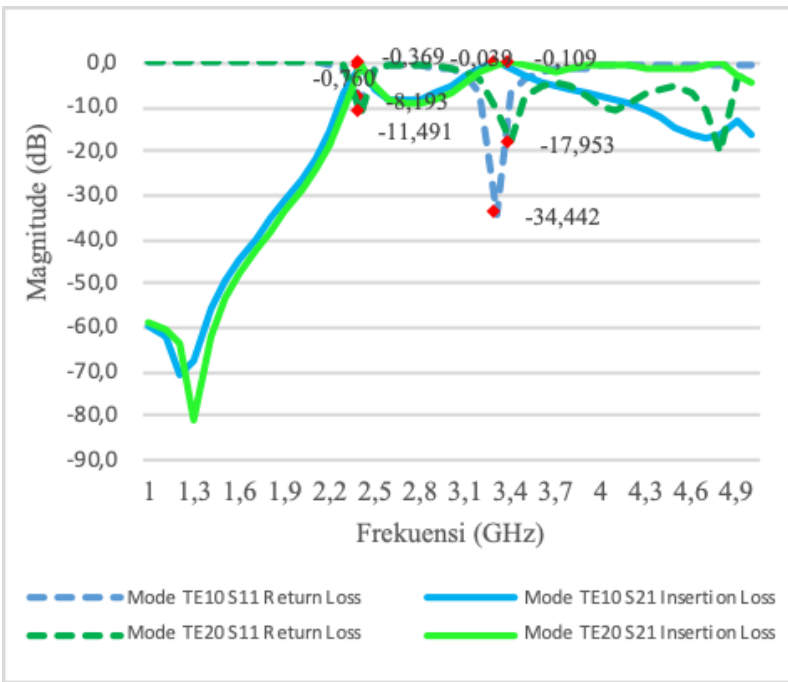

Figure 4. Rectangular waveguide chart using SMA Connectors with position at $65 / 35$ seen in Figures 5 and 6. The design is using SMA connector that is located on $\mathrm{x}$-axis, with rectangular waveguide of $100 \mathrm{~mm}$. The positions of the connector are are shifted to $70 \mathrm{~mm}$ and $30 \mathrm{~mm}$, previously at $65 \mathrm{~mm}$ and $35 \mathrm{~mm}$. The center point is still located at $50 \mathrm{~mm}$. Thus, the distance of the connectors from the center point is $20 \mathrm{~mm}$. Figures 5 and 6 show the results of the $\mathrm{TE}_{10}$ and $\mathrm{TE}_{20}$ modes, respectively.

Figure 7 shows the graph of simulated results by using SMA connectors. In this simulation, the connector position is shifted and the connector position that is based on the $\mathrm{x}$-axis is at the position of 70/30. By performing the connector shift, the obtained values of the parameters are changed, as illustrated in Figure 7 for $\mathrm{TE}_{10}$ mode. The best filter response is at a cut-off frequency of $3.2 \mathrm{GHz}$ with the value of return loss of $-27.764 \mathrm{~dB}$ and an insertion loss of $-0.044 \mathrm{~dB}$. For the $\mathrm{TE}_{20}$ mode, the best filter response is at the cut-off frequency of $3.3 \mathrm{GHz}$ with the value of return loss of $-36.624 \mathrm{~dB}$ and an insertion loss of $-0.034 \mathrm{~dB}$.

The results of the $\mathrm{TE}_{\mathrm{mn}}$ rectangular waveguide mode design by performing a connector shifting position can be seen in Figures 8 and 9. This design is using SMA connector that is located on the $\mathrm{x}$-axis, with a rectangular waveguide length of $100 \mathrm{~mm}$. The positions of the connector are shifted to $75 \mathrm{~mm}$ and $25 \mathrm{~mm}$, previously at $70 \mathrm{~mm}$ and $30 \mathrm{~mm}$. The center point is still located at 50 $\mathrm{mm}$. Thus, the distance of the connectors from the center point is $25 \mathrm{~mm}$. Figures 8 and 9 show the results of the $\mathrm{TE}_{10}$ and $\mathrm{TE}_{20}$ modes, respectively.

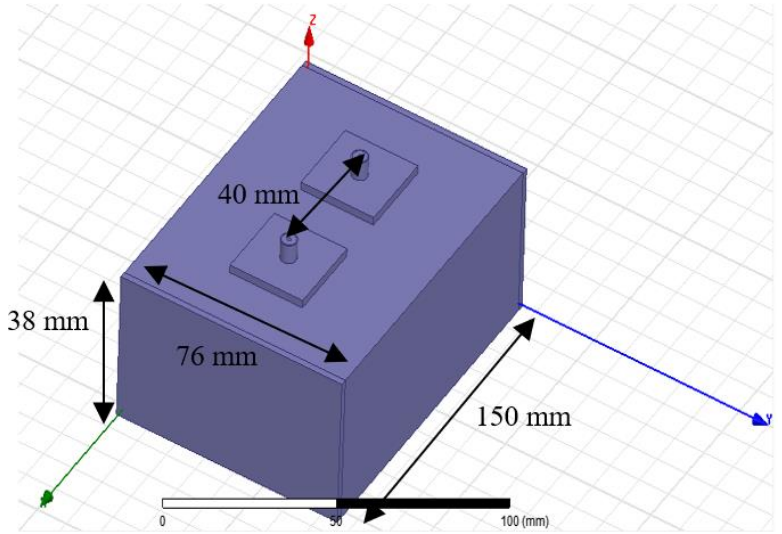

Figure 5. Rectangular waveguide design using connectors with position at 70/30 ( $\mathrm{TE}_{10}$ mode $)$.

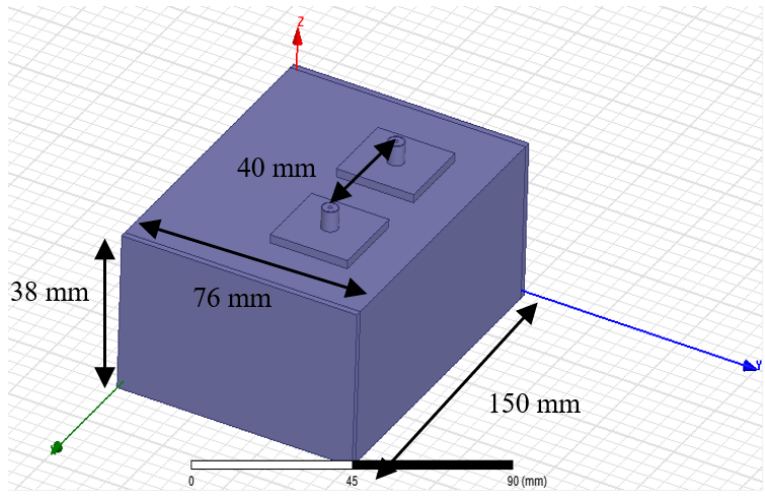

Figure 6. Rectangular waveguide design using connectors with position at $70 / 30\left(\mathrm{TE}_{20}\right.$ mode $)$. 


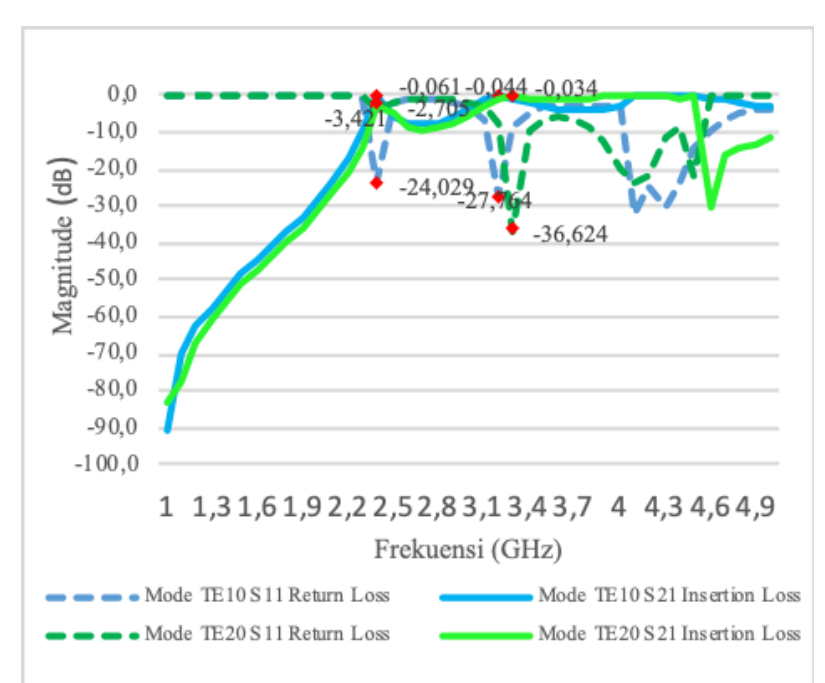

Figure 7. Rectangular waveguide chart using SMA Connectors with position at $70 / 30$

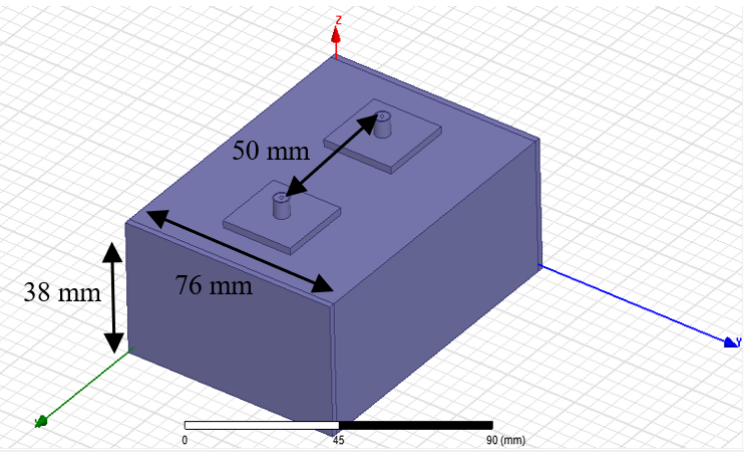

Figure 8. Rectangular waveguide design using connectors with position at 75/25 ( $\mathrm{TE}_{10}$ mode).

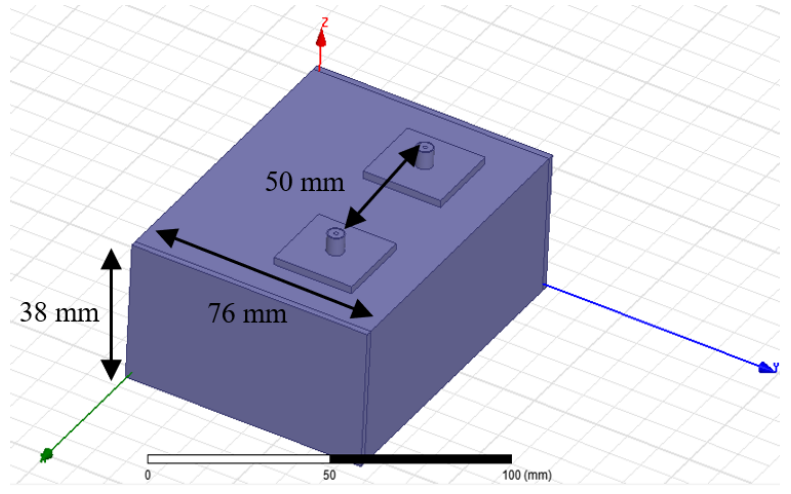

Figure 9. Rectangular waveguide design using connectors with position at 75/25 $\left(\mathrm{TE}_{20}\right.$ mode)

Figure 10 shows the graph of simulated results by using SMA connectors. In this simulation, the connector position is shifted, and the connector position that is based on the $x$-axis is at the position of $75 / 25$. By performing the connector shift, the obtained values of the parameters are changed as illustrated in Figure 10 for $\mathrm{TE}_{10}$ mode. The best filter response is at a cut-off frequency of $3.2 \mathrm{GHz}$ with the value of return loss of $21.658 \mathrm{~dB}$ and an insertion loss of $-0.067 \mathrm{~dB}$. For the $\mathrm{TE}_{20}$ mode, the best filter response is at the cut-off frequency of $3.3 \mathrm{GHz}$ with the value of return loss of $26.062 \mathrm{~dB}$ and an insertion loss of $-0.049 \mathrm{~dB}$.
The results of the design of the $\mathrm{TE}_{\mathrm{mn}}$ rectangular waveguide mode by performing a connector shifting position can be seen in Figures 11 and 12. The design is using SMA connector that is located on the $\mathrm{x}$-axis, with a rectangular waveguide length of $100 \mathrm{~mm}$. The positions of the connector are shifted to $80 \mathrm{~mm}$ and $20 \mathrm{~mm}$, previously at $75 \mathrm{~mm}$ and $25 \mathrm{~mm}$. The center point is still located at $50 \mathrm{~mm}$. Thus, the distance of the connectors from the center point is $30 \mathrm{~mm}$. Figures 11 and 12 show the results of the $\mathrm{TE}_{10}$ and $\mathrm{TE}_{20}$ modes, respectively.

Figure 13 shows the graph of simulated results by using SMA connectors. In this simulation, the connector position is shifted and the connector position that is based on the $\mathrm{x}$-axis is at the position of $80 / 20$. By performing the connector shift, the obtained values of the parameters are changed, as illustrated in Figure 13. For $\mathrm{TE}_{10}$ mode, this configuration produces the best filter response.

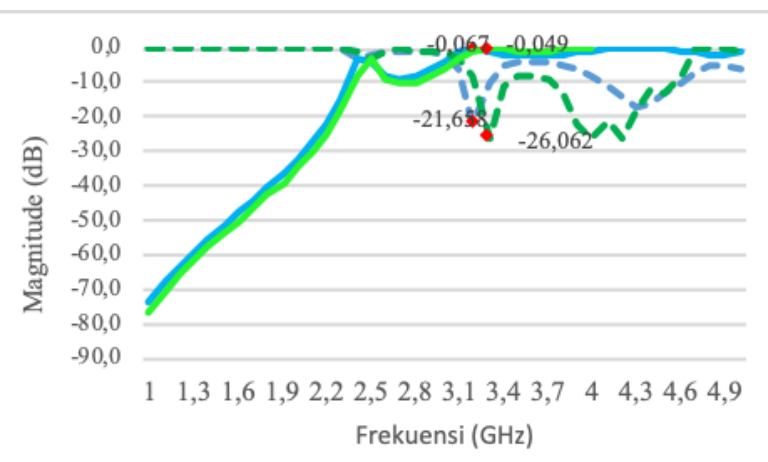

- - Mode TE10S11 Return Loss

- - Mode TE20S11 Return Los

Mode TE10 S 21 Insertion Loss Mode TE20S21 Insertion Loss

Figure 10. Rectangular waveguide chart using SMA Connector with position $75 / 25$.

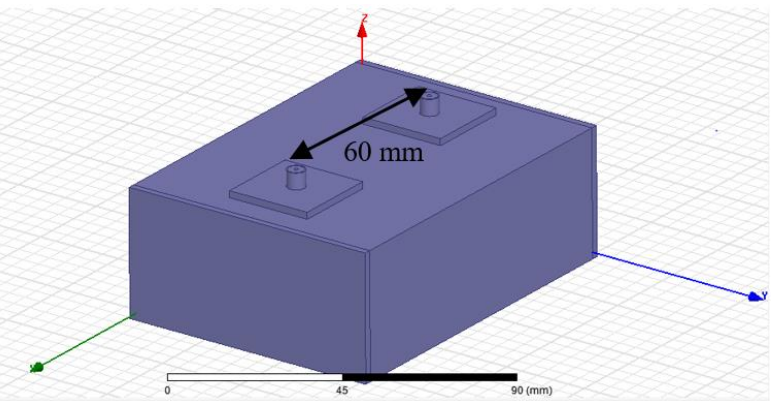

Figure 11. Rectangular waveguide design using connector with position 80/20 Mode TE10

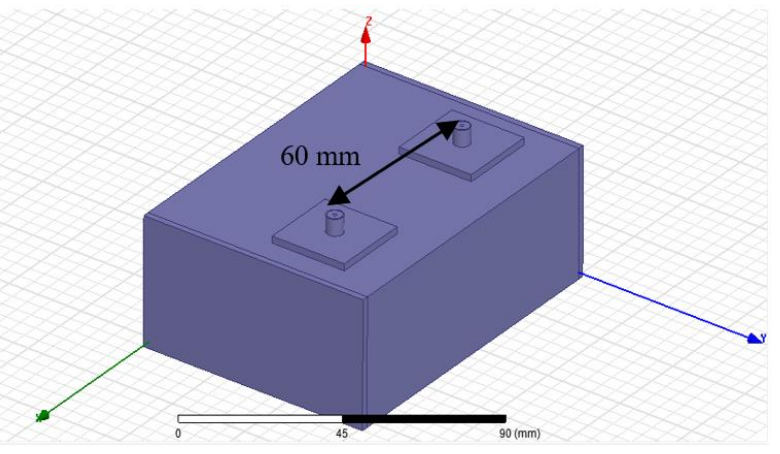

Figure 12. Rectangular waveguide design using connectors with position at $80 / 20\left(\mathrm{TE}_{20}\right.$ mode $)$. 


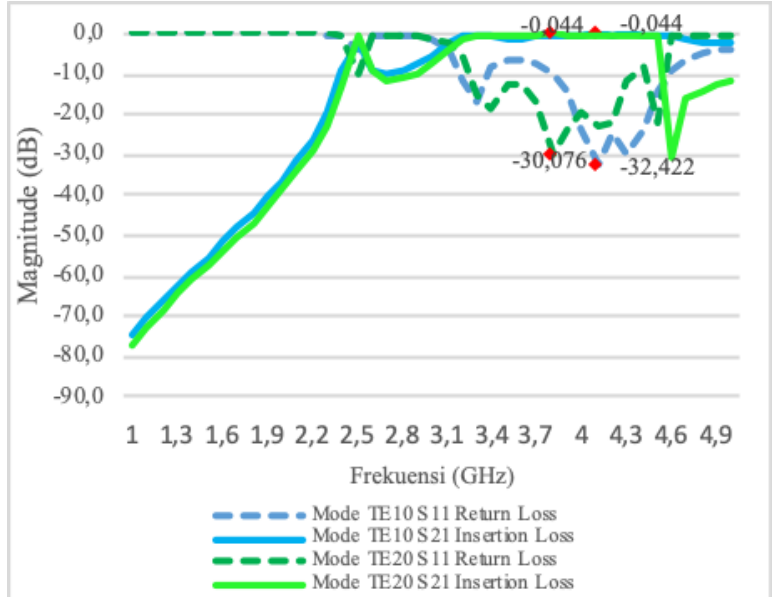

Figure 13. Rectangular waveguide chart using SMA Connectors with position at $80 / 20$

The cut-off frequency is shifted from $3.2 \mathrm{GHz}$ to $4.1 \mathrm{GHz}$ with the value of return loss of $-32.422 \mathrm{~dB}$ and an insertion loss of $-0.044 \mathrm{~dB}$. For $\mathrm{TE}_{20}$ mode, the filter response is also shifted, from previously at a cut-off frequency of $3.3 \mathrm{GHz}$ to $3.8 \mathrm{GHz}$, with a return loss of $30.070 \mathrm{~dB}$ and an insertion loss of $-0.044 \mathrm{~dB}$. For this simulation scenario, although the obtained value of the return loss and insertion loss parameters are very well compared to the previous simulation results, the cut-off frequency for both modes shifted from the expected frequency.

The results of the design of the $\mathrm{TE}_{\mathrm{mn}}$ rectangular waveguide mode by performing a connector shifting position can be seen in Figures 14 and 15. The design is using SMA connector that is located on the $\mathrm{x}$-axis, with a rectangular waveguide length of $100 \mathrm{~mm}$. The positions of the connector are shifted to $85 \mathrm{~mm}$ and $15 \mathrm{~mm}$, previously at $75 \mathrm{~mm}$ and $25 \mathrm{~mm}$. The center point is still located at $50 \mathrm{~mm}$. Thus, the distance of the connectors from the center point is $35 \mathrm{~mm}$. Figures 14 and 15 show the results of the $\mathrm{TE}_{10}$ and $\mathrm{TE}_{20}$ modes, respectively.

Figure 16 shows the graph of simulated results by using SMA connectors. In this simulation, the connector position is shifted, and the connector position that is based on the x-axis is at the position of $85 / 15$. By performing the connector shift, the obtained values of the parameters are changed, as illustrated in Figure 16 for $\mathrm{TE}_{10}$ mode. The best filter response is at a cut-off frequency of $3.4 \mathrm{GHz}$ with the value of return loss of $21.971 \mathrm{~dB}$ and an insertion loss of $-0.263 \mathrm{~dB}$. For the $\mathrm{TE}_{20}$ mode, the best filter response is at the cut-off frequency of $3.5 \mathrm{GHz}$ with the value of return loss of $28.718 \mathrm{~dB}$ and an insertion loss of $-0.045 \mathrm{~dB}$.

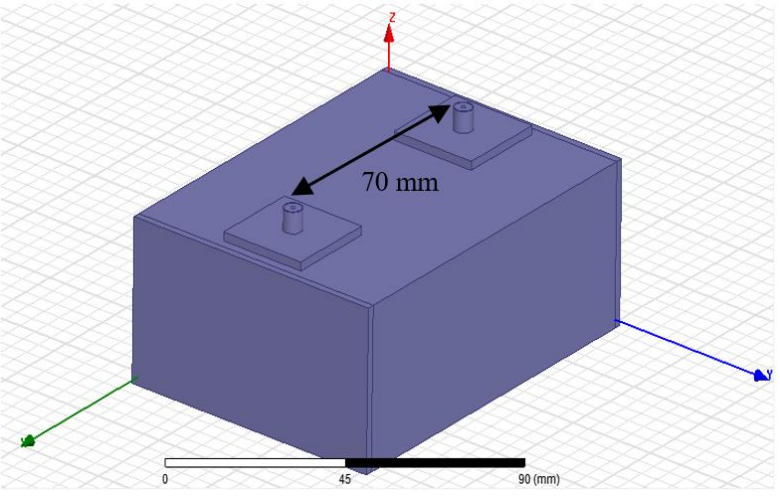

Figure 14. Rectangular waveguide design using connectors with position at $85 / 15\left(\mathrm{TE}_{10}\right.$ mode)

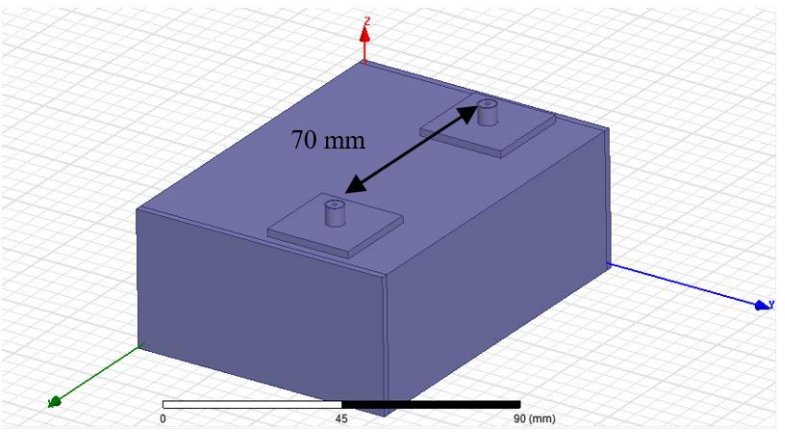

Figure 15. Rectangular waveguide design using connectors with position at $85 / 15\left(\mathrm{TE}_{20}\right.$ mode)

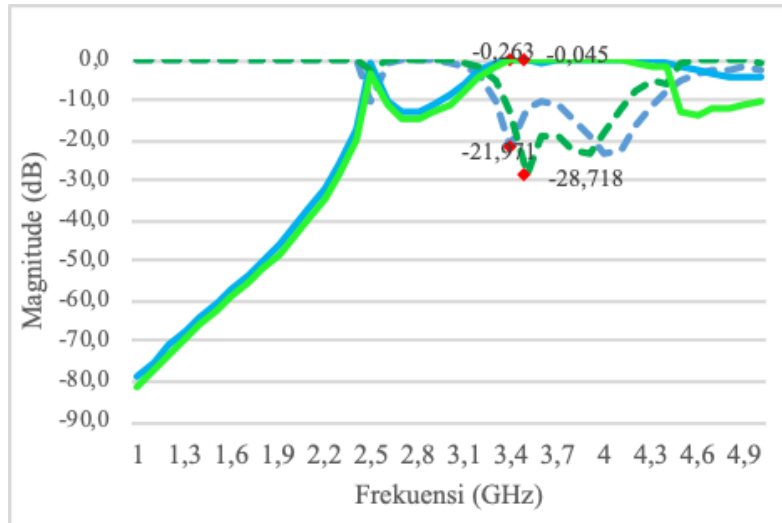

- - Mode TE10 11 Return Loss

- - Mode TE20 111 Return Loss

Mode TE10 S21 Insertion Loss

Figure 16. Rectangular waveguide chart using SMA Connectors with position at $80 / 20$.

\section{Analysis of $\mathbf{T E}_{\mathrm{mn}}$ Mode Rectangular Waveguide Design Using SMA Connector}

After the optimization of the design, the simulation results are then analyzed. The best design is fabricated and then is measured using Vector Network Analyzer (VNA). Figure 17 shows a comparison chart of all simulated $\mathrm{TE}_{10}$ mode rectangular waveguide results by shifting the connector position. Because the smaller the value of the return loss parameters is the better, the best return loss value is in the position of connector $65 / 35$ and connector $80 / 20$. However, for the connector position at $80 / 20$, the parameter of the cut-off frequency is at 4.1 $\mathrm{GHz}$, and the value is not in the expected frequency range 
of 3.3-3.5 GHz. Whereas, for connector position 65/35, the parameter of the cut-off frequency is at $3.3 \mathrm{GHz}$ and the value is at the expected frequency range. In conclusion, for the $\mathrm{TE}_{10}$ mode rectangular waveguide, the best performance is obtained when the connector is at position 65/35.

Figure 18 shows a comparison chart of all simulated $\mathrm{TE}_{20}$ mode rectangular waveguide results by shifting the connector position. The best return loss value is at the connector position 70/30. However, as seen in Figure 18, for the position 70/30 connector, in one bandwidth there are three parameters of the cut-off frequency passed in the filter response. The required filter response in one bandwidth can only miss a slight cut-off frequency. For the $85 / 15$ connector position, in one bandwidth simply skip two parameters of the cut off frequency and the best value at the position of the cut off frequency at $3.5 \mathrm{GHz}$.

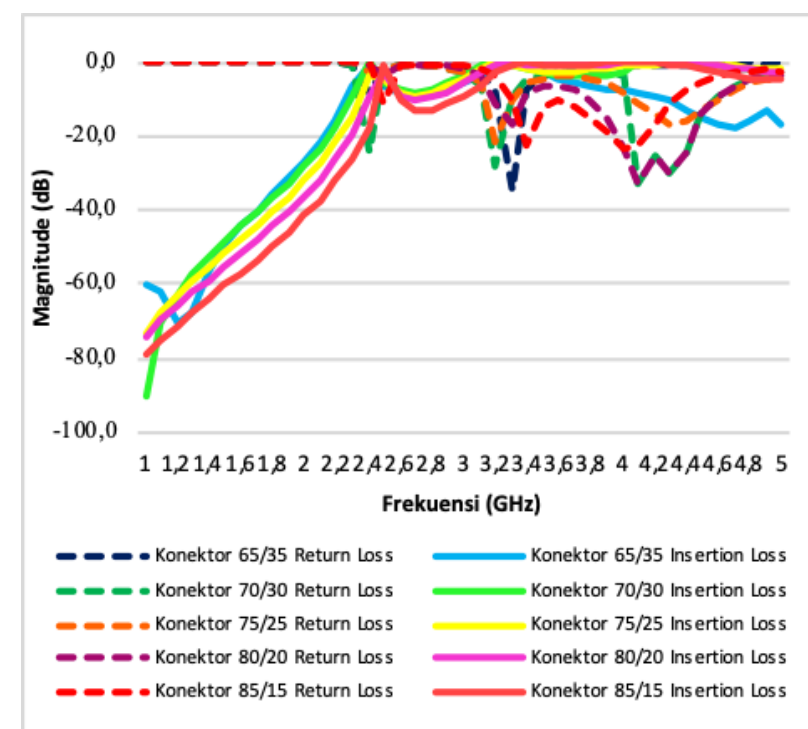

Figure 17. Comparison chart of simulated result shifting connectors on $\mathrm{TE}_{10}$ mode.

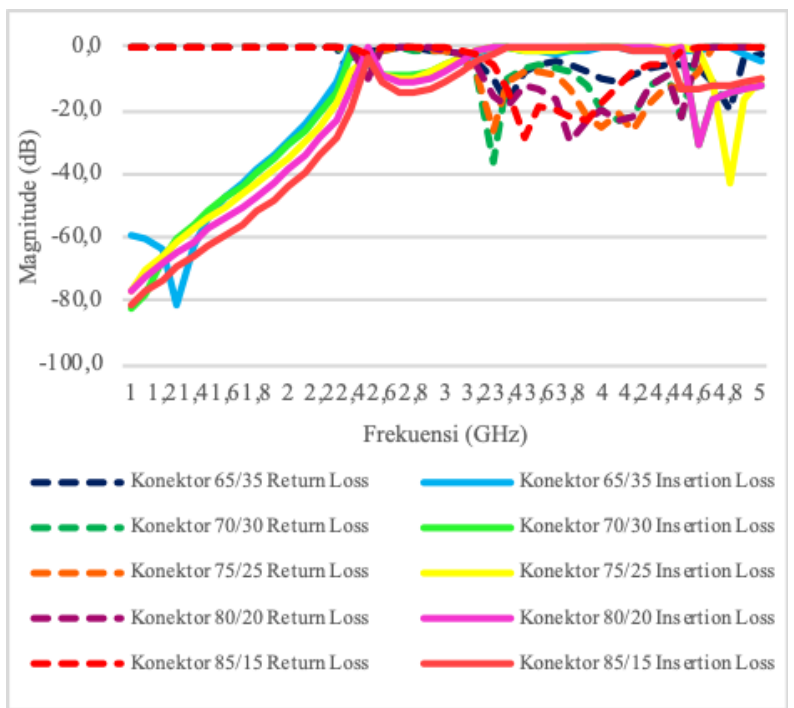

Figure 18. Comparison chart of simulated result shifting connectors on $\mathrm{TE}_{20}$ mode.

\section{Power Field Raised in Simulation}

Figure 19 shows the simulated electric field (E) in $\mathrm{TE}_{10}$ mode rectangular waveguide. The highest value of electric field intensity is at the point of axis $y=36 \mathrm{~mm}$ (half of the waveguide width $72 \mathrm{~mm}$ ) and $\mathrm{z}=17 \mathrm{~mm}$ (half of the waveguide height $34 \mathrm{~mm}$ ), where the intensity value of $E=27.3 \mathrm{~V} / \mathrm{M}$. Figure 20 shows the simulated electric field (E) in $\mathrm{TE}_{20}$ mode rectangular waveguide. The highest value of electric field intensity is at the point of axis $\mathrm{y}=48 \mathrm{~mm}(2 / 3$ of $72 \mathrm{~mm}$ width $)$ and $\mathrm{z}=17 \mathrm{~mm}$ (1/2 of $34 \mathrm{~mm}$ width), where the intensity value $\mathrm{E}=350$ V/M.

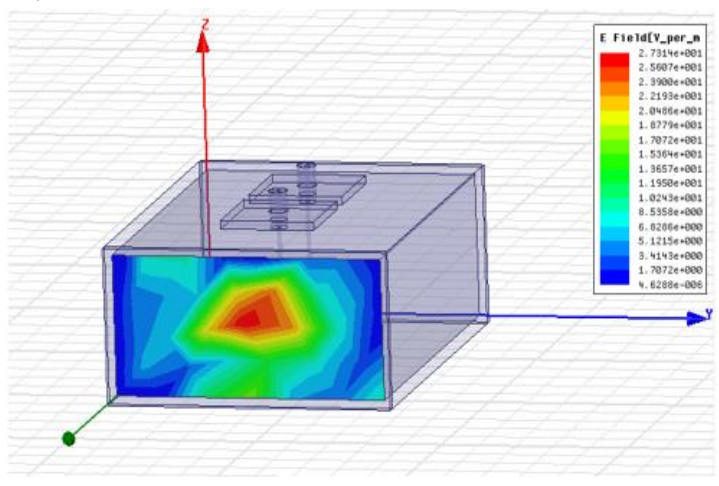

Figure 19. Simulated electric field in $\mathrm{TE}_{10}$ mode.

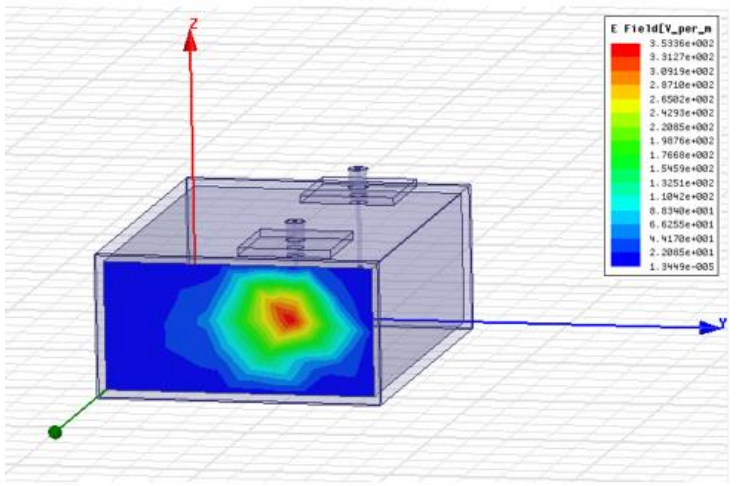

Figure 20. Simulated electric field in $\mathrm{TE}_{20}$ mode.

\section{E. Design Realization}

The fabricated rectangular waveguide that is based on the simulation can be seen in Figures 21 and 22.

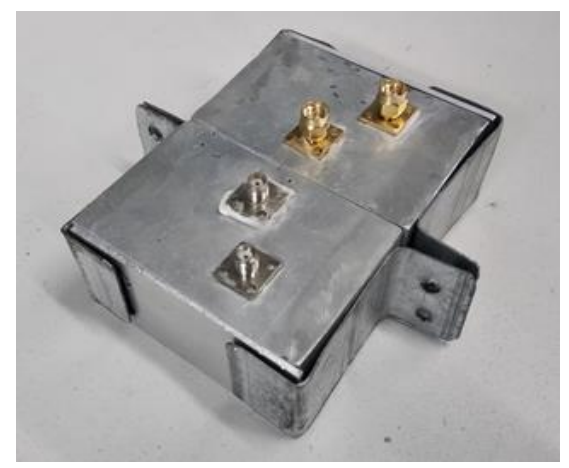

Figure 21. Realization of the design. 
TABLE 1

COMPARISON RESULT BETWEEN MEASUREMENT AND SIMULATION

\begin{tabular}{|c|c|c|c|c|c|c|c|c|c|c|c|}
\hline \multicolumn{6}{|c|}{ Simulation Results (65/35) } & \multicolumn{6}{|c|}{ Measurement Results (with VNA) } \\
\hline \multicolumn{3}{|c|}{ TE $_{10}$ Mode } & \multicolumn{3}{|c|}{ TE $_{20}$ Mode } & \multicolumn{3}{|c|}{ TE $_{10}$ Mode } & \multicolumn{3}{|c|}{ TE 20 Mode } \\
\hline $\mathrm{F}(\mathrm{GHz})$ & $\mathrm{S}_{11}(\mathrm{~dB})$ & $\mathrm{S}_{21}(\mathrm{~dB})$ & $\mathrm{F}(\mathrm{GHz})$ & $\mathrm{S}_{11}(\mathrm{~dB})$ & $\mathrm{S} 21(\mathrm{~dB})$ & $\mathrm{F}(\mathrm{GHz})$ & $\mathrm{S}_{11}(\mathrm{~dB})$ & $\mathrm{S} 21(\mathrm{~dB})$ & $\mathrm{F}(\mathrm{GHz})$ & $\mathrm{S}_{11}(\mathrm{~dB})$ & $\mathrm{S}_{21}(\mathrm{~dB})$ \\
\hline 3.3 & 34 & 0.39 & 3.4 & 17 & 0.109 & 3.2 & 18 & 2.7 & 3.2 & 5.89 & 4.31 \\
\hline
\end{tabular}

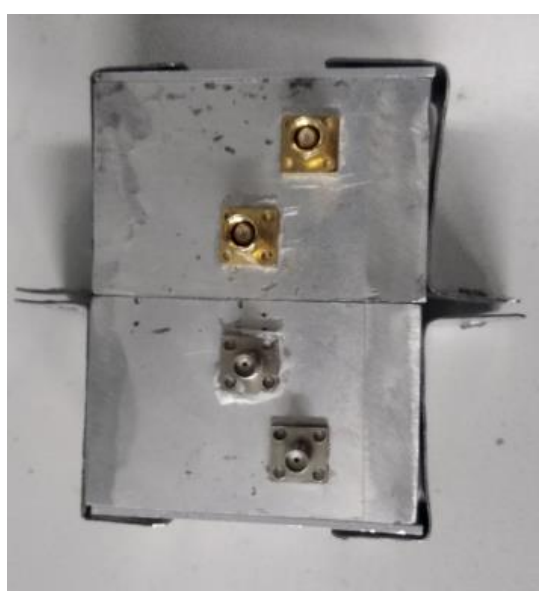

Figure 22. Realization of the design (top view).

After fabrication, the rectangular waveguide is then measured using Vector Network Analyzer (VNA) for both modes ( $\mathrm{TE}_{10}$ and $\left.\mathrm{TE}_{20}\right)$. Based on the measurement results for the $\mathrm{TE}_{10}$ mode, the cut-off frequency is at 3.2 $\mathrm{GHz}$ with the value of the return loss of $-18.73 \mathrm{~dB}$ and an insertion loss of $-2.70 \mathrm{~dB}$. To compare, the simulated results at connector position 65/35 are: cut-off frequency $=3.3 \mathrm{GHz}$, return loss $-34.442 \mathrm{~dB}$, and insertion loss = $0.039 \mathrm{~dB}$. This means that the performance of the fabricated device experiences a cut-off frequency shifting from $3.3 \mathrm{GHz}$ to $3.2 \mathrm{GHz}$. Nevertheless, the values of the return loss and insertion loss are satisfactory.

The measured cut-off frequency for $\mathrm{TE}_{20}$ mode is at a frequency of $3.2 \mathrm{GHz}$ with the value of the return loss of $-5.89 \mathrm{~dB}$ and an insertion loss of $-4.31 \mathrm{~dB}$. Because the value of the return loss is below than $-3 \mathrm{~dB}$ and the value of the insertion loss is above $-10 \mathrm{~dB}$, it can be said that the obtained measurement results for the $\mathrm{TE}_{20}$ mode is not better than the simulation. The simulated results for the $\mathrm{TE}_{20}$ mode are better than the measured results with a cut-off frequency of $3.5 \mathrm{GHz}$, a return loss of $-28.718 \mathrm{~dB}$ and an insertion loss of $-0.045 \mathrm{~dB}$.

Based on the comparison of the simulated and measurement results, there is a difference in the value of the parameters. The discrepancy is due to the dimensional changes in the manufacturing process (device fabrication). The fabrication process needs a high level of accuracy, and it is difficult to obtain a dimension that corresponds to the simulation. Results comparison between measurement and simulation can be seen in Table 1.

\section{CONCLUSION}

Based on the simulation of $\mathrm{TE}_{10}$ mode rectangular waveguide using connectors, the best performance obtained at the position of $65 / 35$ (30 $\mathrm{mm}$ distance between connectors) with a cut-off frequency of $3.3 \mathrm{GHz}$, a return loss of $-34.442 \mathrm{~dB}$ and an insertion loss of -0.039 $\mathrm{dB}$. While, for the simulated $\mathrm{TE}_{20}$ mode rectangular waveguide using connectors, the best results are obtained at the position of 85/15 (distance between connectors = $70 \mathrm{~mm}$ ) with a cut off frequency of $3.5 \mathrm{GHz}$, a return loss of $-28.718 \mathrm{~dB}$ and an insertion loss of $-0.045 \mathrm{~dB}$.

The measured results of $\mathrm{TE}_{10}$ mode rectangular waveguide with the connectors position adjacent to the filter response is at a cut-off frequency of $3.2 \mathrm{GHz}$ with the value of the return loss of $-18.73 \mathrm{~dB}$ and an insertion loss of $-2.70 \mathrm{~dB}$. Menwhile, the measured result of the $\mathrm{TE}_{20}$ mode rectangular waveguide TE20 is at a cut-off frequency of $3.2 \mathrm{GHz}$ with the value of the return loss of $-5.89 \mathrm{~dB}$ and an insertion loss $-4.31 \mathrm{~dB}$.

\section{ACKNOWLEDGEMENT}

We thank our fellow in Laboratory of Electrical Engineering Universitas Jenderal Achmad Yani which has provided assistance in this research.

\section{REFERENCES}

[1] A. E. Karbowiak, "Microwave aspects of waveguides for longdistance transmission," in Proc. IEE Part C Monogr., vol. 105, no. 8 , pp. $360,1958$.

[2] S. E. Miller, "Waveguide as a communication medium," Bell Syst. Tech. J., vol. 33, no. 6, pp. 1209-1265, 1954.

[3] E. F. Da Silva, J. S. Rocha, P. R. Lins, S. D. Da Nóbrega, and M. S. De Alencar, "Characterization of electromagnetic radiation absorber materials," in Proc. SBMO/IEEE MTT-S Int. Microwave Optoelectronics Conf., 2005, pp. 326-329.

[4] G. Sindura, K. Ram Prakash, and P. Salil, "Control of electromagnetic waves through electromagnetic shielding," in Proc. 2011 Int. Conf. Emerging Trends Elect. Comput. Technology, 2011, pp. 448-452.

[5] A. Sabouni, S. Noghanian, and L. Shafai, "Transverse electric and magnetic components of field measurement for microwave breast cancer imaging," in Proc. 2010 14th Int. Symp. Antenna Tech. Appl. Electromagnetics American Electromagnetics Conf., 2010, pp. 1-4.

[6] D. F. Cui, Z. H. Chen, Z. Y. Yuan, Y. L. Zhou, H. B. Lu, and G. $Z$. Yang, "Transverse electric and transverse magnetic active intersubband transitions in GaAs/AlGaAs step quantum well," in Proc. IEEE Lasers Electro-Optics Soc. $19947^{\text {th }}$ Annu. Meeting, 1994, vol. 1, pp. 182-183.

[7] M. Omar, N. A. M. Zin, Z. I. A. Latiff, and N. A. Ismail, "Review on design of on chip band pass filter for radio frequency applications," in Proc. 2016 7th IEEE Control Syst. Graduate Research Colloquium, 2016, pp. 148-152.

[8] D. Selvathi and M. Pown, "Design of band pass filter using active inductor for RF receiver front-end," in Proc. 2014 Int. Conf. Commun. Network Technologies, 2014, pp. 296-301. 
[9] N. Chakrabarti, S. Kalra, S. Saxena, and M. R. Tripathy, "Ultrawideband antenna for a ground penetrating radar," in Proc. 2016 Thirteenth Int. Conf. Wireless Optical Commun. Networks, 2016.

[10] F. Parrini et al., "ULTRA: Wideband ground penetrating radar," in Proc. 2006 European Radar Conf., 2006, pp. 182-185.

[11] Y. $\mathrm{Xu}$ et al., "Design and test of broadband rectangular waveguide TE10 to circular waveguide TE21 and TE01 mode converters," IEEE Trans. Electron. Devices, vol. 66, no. 8, pp. 3573-3579, Aug. 2019.

[12] H. S. Wu and C. K. C. Tzuang, "Artificially integrated synthetic rectangular waveguide," IEEE Trans. Antennas Propag., vol. 53, no. 9, pp. 2872-2881, Sep. 2005.

[13] F. Teberio et al., "Rectangular waveguide filters with meandered topology," IEEE Trans. Microw. Theory and Tech., 2018, vol. 66, no. 8 , pp. $3632-3643$.

[14] Z. Yu, "The miniaturization of rectangular waveguide," in Proc. 2010 Int. Conf. Microw. Millimeter Wave Tech., 2010, pp. 1845 1848. 